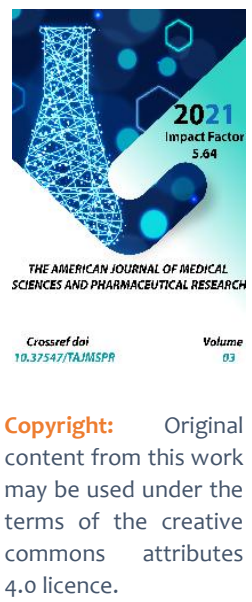

\section{Comparative Characteristics Of Surgical Treatment Methods For Patients With Nasal Septum Deviation}

\author{
Ulugbek Saidakramovich Khasanov \\ Department Of Otolaryngology And Stomatology Tashkent Medical Academy, Uzbekistan \\ Otabek Rustamovich Khayitov \\ Department Of Otolaryngology And Stomatology Tashkent Medical Academy, Uzbekistan \\ Jamolbek Abdukakharovich Djuraev \\ Department Of Otolaryngology And Stomatology Tashkent Medical Academy, Uzbekistan
}

\title{
ABSTRACT
}

The methodological recommendation presents a comparative characteristic of surgical methods of treatment of deviation of the nasal septum. Deviation of the nasal septum occurs in the cartilaginous and bone regions, both separately and in both simultaneously. Deformations can be of different nature (bend, crest, spike) and localization. They are one and two-sided. Spines and ridges are more often localized at the junction of the quadrangular cartilage and the perpendicular plate, ploughshare, and the crest of the upper jaw ("growth zone"). Less often, the upper sections of the perpendicular plate and the rear section of the ploughshare are deformed. For traumatic deformities, characteristic bends with acute angles. Often, after injury, there is a displacement of the anterior edge of the quadrangular cartilage in the form of its subluxation. The deformation of the cartilaginous part of the external nose in patients under our supervision was caused by the deviation of the nasal septum. There were no patients with deformity of the nose who would have been indicated for surgical intervention on the cartilage of the external nose. When correcting the deformity of the external nose in patients, we used an exclusively closed technique of intervention with a transseptal access, and, if necessary, access under the upper lip, which allowed us to obtain a good cosmetic effect and minimize traumatic complications after surgery.

\section{KEYWORDS}

Deviation of the nasal septum, submucosal resection, septoplasty, quadrangular cartilage. 


\section{INTRODUCTION}

Despite a number of reports on the curvature of the nasal septum in newborns, it should be noted that in early childhood, its deformity, as a rule, is absent $[1,2,3,4]$. The frequency of this pathology of the nasal septum increases in proportion to the age of the person, and is much more common in boys. So, among 180 children with deformities of the nasal septum aged 5 to 15 years who were examined and treated by the authors, there were $81 \%$ of boys, and only $19 \%$ of girls.

Curvature of the nasal septum occurs in the cartilaginous and bone regions, both separately and in both simultaneously $[5,6,7]$. Deformations can be of different nature (bend, crest, spike) and localization. They are one and two-sided. Spines and ridges are more often localized at the junction of the quadrangular cartilage and the perpendicular plate, ploughshare, and the crest of the upper jaw ("growth zone"). Less often, the upper sections of the perpendicular plate and the rear section of the ploughshare are deformed. For traumatic deformities, characteristic bends with acute angles [4]. Often, after injury, there is a displacement of the anterior edge of the quadrangular cartilage in the form of its subluxation.

Long-term difficulty in breathing, which occurs in early childhood, leads to a violation of the development of the face, namely, it becomes elongated and narrow, flattened from the sides, the hard palate forms a high Gothic palate, the alveolar arch lengthens, the development of teeth is disturbed, the lower jaw droops, the mouth constantly half open, nasolabial folds are smoothed out $[8,9,10,11]$.

Submucosal resection of the nasal septum began to be performed since 1882 by Ingals in Chicago and Hartmann, Peterson, Krieg in
Germany, and it was performed in children (cited by G.I. Piskunov and S.I. Piskunov, 2006). At the beginning of the twentieth century, submucosal resection of the nasal septum was widely used according to the methods of Freer (1902) and Killian (1904).

I.A. Voyachek and M.G. Dangulov developed a number of economical techniques for operations on the nasal septum: "mobilization", "partial submucosal resection", "circular resection", "redressing", "disc method". These techniques made it possible to perform operations on the nasal septum, almost completely preserving the support structures of the nose. Technique for performing septoplasty according to V.Y. Voyachek is as follows. First, a skin incision is made in the anterior part of the septum, soft tissues are exfoliated from the curvature point closest to the nostril on the convex side, and the quadrangular cartilage is opened without damaging the mucous membrane on the other side of the septum. Next, several cuts are made in the cartilage in the form of a circle or polygon, thereby part of the cartilage becomes mobile and hangs on the mucous membrane of the opposite side. Then, with the help of a raspator, a similar movable disc is formed from the opposite side in deeper bone regions [12]. In case of curvatures in the lower parts, the mucous membrane peels off from the lowermost part of the curvature and is separated from the bottom of the nose with a chisel so that a disc is formed connected with the soft tissues of one side. If necessary, additional discs are formed, which make the septum pliable, and it is easily installed in the middle position $[13,14,15]$.

Cottle outlined his method for conservative septoplasty. The technique for performing 
conservative septoplasty is as follows: an autopsy is performed on the left, stepping back 1-2 $\mathrm{mm}$ from the caudal edge of the cartilage, the perichondrium dilator exfoliates along the entire length of the septum to the anterior wall of the sphenoid sinus, without going down below the wing of premaxili (this is how the anteroposterior left upper tunnel is formed). Then, along the base of the septum under the wing of the premaxil, an anteroposterior inferior tunnel is formed. The connective tissue membrane between the two tunnels is dissected and a wide access is formed to the left surface of the septum. If this gives access to distortion, it is corrected. The cartilaginous septum is separated from the premaxil. The cartilage is dissected perpendicularly, leaving $2-3 \mathrm{~cm}$ from the caudal edge, to the opposite side, without injuring the mucous membrane of the opposite side [16]. Correction of the bony part of the septum is performed. To form a wider access, the right anteroposterior upper and lower tunnels are formed, which are connected to each other $[17,18,19]$. In this case, only curved parts are removed with minimal resection.

\section{MATERIAL AND METHODS}

In accordance with the purpose of the study and to fulfill the assigned tasks, clinical studies were carried out in 160 patients with curvature of the nasal septum, who were hospitalized in the ENT department of "QO'QON DUNYO JAVOHIRI" for 2017-2020. All patients underwent a comprehensive examination, including the collection of complaints, examination of the ENT organs, endoscopy of the nasal cavity and X-ray examinations.

\section{RESULTS AND DISCUSSION}

Preoperative preparation of patients with nasal septum curvature. When preparing a patient for surgery, the general somatic state was assessed, blood pressure, body temperature, clinical and biochemical blood and urine tests were checked. Previous illnesses were taken into account (the operation was performed 1 month after ARVI and 6 months after childhood infections). A thorough sanitation of foci of chronic infection was carried out. They made sure that there were no inflammatory processes on the skin of the face.

Patients with chronic decompensated tonsillitis and chronic adenoiditis underwent tonsillectomy and adenotomy, and septoplasty was planned no earlier than a month later. If the parents refused to have tonsillectomy, conservative treatment of chronic tonsillitis was carried out, and a month after improvement of the condition, septoplasty was performed. If patients with nasal septum curvature and grade II-III adenoid vegetations were present, septoplasty and adenotomy were performed simultaneously.

The operation began with an adenotomy. This sequence promoted hemostasis in the nasal part of the pharynx. In children with insignificant deformity of the nasal septum or under the age of 7 years, as a rule, adenotomy was performed and subsequently, the dynamics of nasal breathing was assessed over the next 1-2 years. The tactics regarding septoplasty and the timing of its implementation were determined individually. 
We performed reconstructive surgery on the nasal septum, depending on the indications, from the age of 7. However, in some cases, given the degree of difficulty in nasal breathing and the possibility of developing diseases that may be caused by a curvature of the nasal septum, septoplasty should be recommended for younger children. In this case, it is necessary to focus not only on the patient's age, but also on existing or possible pathological changes in the body in the future.

In patients with curvature of the nasal septum, deformity of the external nose, septoplasty and rhinoplasty were simultaneously planned.

In the presence of hypertrophy of the turbinates on the side opposite to the curvature of the nasal septum, it was planned to perform ultrasonic disintegration of the turbinate after the completion of septoplasty.

1-2 weeks prior to septoplasty, ascorutin and calcium gluconate were prescribed to increase blood clotting. Dicinone was administered two hours before the operation. Surgical intervention was performed under general anesthesia.

Techniques for surgical interventions on the nasal septum.

According to our method, after premedication, under general anesthesia with infiltrative anesthesia of the nasal septum mucosa using Sol. Novocaini $1 \%-10.0$ on a deviated nasal septum. With the help of a scalpel, retreating $0.5 \mathrm{~cm}$ from the entrance and from the back of the nose, a vertical incision is made in the curved part of the nasal septum, and then the incision is extended in the horizontal direction. In this case, the curved part of the mucosa, submucosa and cartilage are dissected simultaneously, without separating the mucous membrane from the perichondrium and without damaging the perichondrium and the mucous membrane of the opposite side. Then the nasal cavity is inserted into the branch of Killian's nasal speculum and redressing is done on the opposite side of the nose and anterior nasal tamponade is performed or a polyethylene tube (splint) is placed. After 2 days, the tampons are removed, and the tube is left for 7-10 days.

With the help of ultrasound, the technique is the same and an ultrasonic scalpel is used. According to our technique, the incision is prolonged and horizontally, this facilitates the mobilization of the septum quite easily and painlessly. In addition, an unseparated mucous membrane with cartilage is not observed after an operation complication such as perforation of the mucous membrane of the nasal septum, hematoma of the nasal septum, and others. Wound healing occurs rather quickly. The operation time is short. Using an ultrasonic scalpel, the operation is performed with little trauma and little blood flow. Bed days after surgery are shortened by 2-4 days. Our method of operation can be performed simultaneously on the nasal septum and paranasal sinuses (endonasal sinusitis, frontotomy), on the ocular canal (dacryocystorhinostomy), nasopharynx (adenotomy) and ear (antrotomy, mastoidotomy).

Correction of curvature of the bony structures of the nasal septum.

The main method for correcting the curvature of the nasal septum in the bony part was its thinning up to $1-2 \mathrm{~mm}$ in thickness with the preservation of only the bone in the sagittal 
plane, drawn after redressing the midline. The operation was performed using a drill using appropriate burs and cutters, or a flat chisel. This technique allows in most cases to eliminate bone deformity and to preserve the

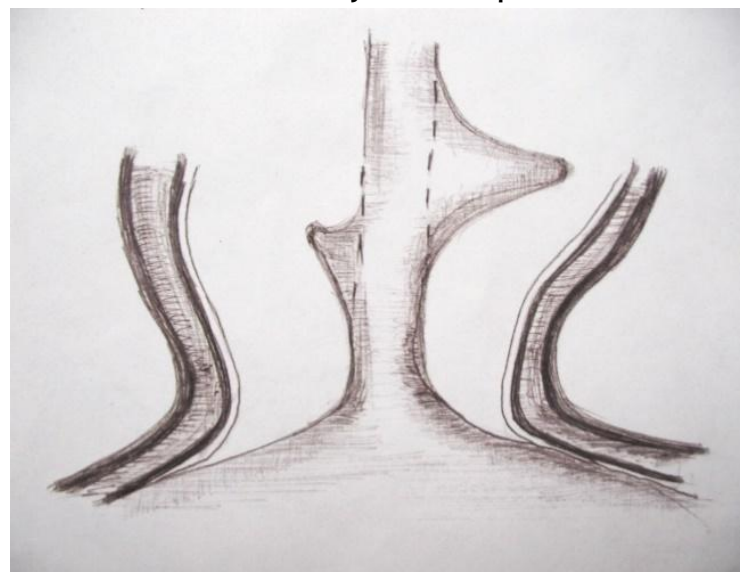

a bony base of the nasal septum in the sagittal plane passing through the midline (Fig. 3.1).

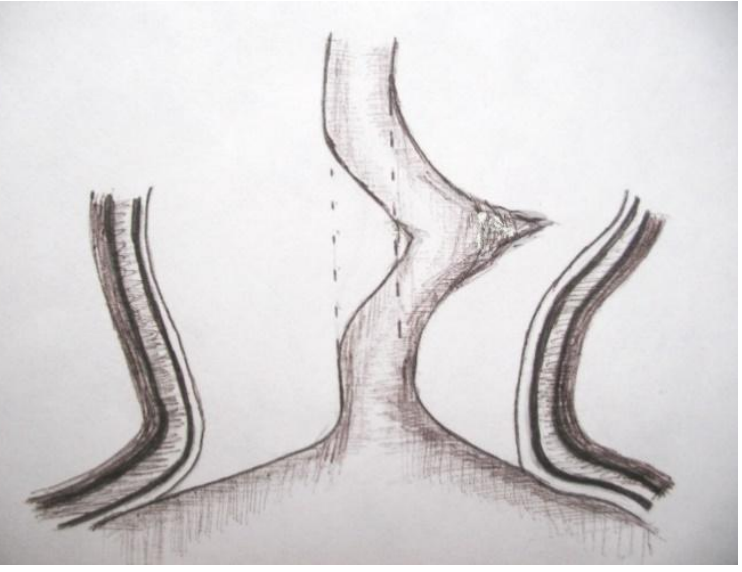

6

Fig. 3.1. Correction scheme for bony deformity of the nasal septum (using a drill).

With a slight curvature of the ploughshare and other bony components of the nasal septum, as a result of the operation, as a rule, the preservation of the bone base in the sagittal plane, drawn through the midline, is achieved in all areas (Fig. 3.1 a). In the presence of spines and ridges with concave areas of the bone on the opposite side, after removal of the curved areas of the bone in the bone plate located in the midline, sometimes there were small holes (Fig. 3.1 b), which did not affect the results of treatment, since the bone framework was stored partitions.

If it was impossible to restore the bony part of the nasal septum while preserving its own tissues, for example, with a pronounced deviation of the share from the midline, the share was removed, and a plate made of quadrangular cartilage was implanted in its place. With a sufficient thickness of the cartilage, partial resection was performed, with the exception of areas in the dorsal and caudal regions with a width of at least $1 \mathrm{~cm} .2$ or 3 plates of uniform thickness were made from the removed cartilage using a special device (Fig. 3.2), and the cartilage was cut in the sagittal plane. In one case, cartilaginous plates were made from the autoreb of a patient who had a curvature of the nasal septum in the bony part, complete destruction of the quadrangular cartilage with drooping of the nasal tip, and saddle deformity of the external nose after an abscess of the nasal septum. 


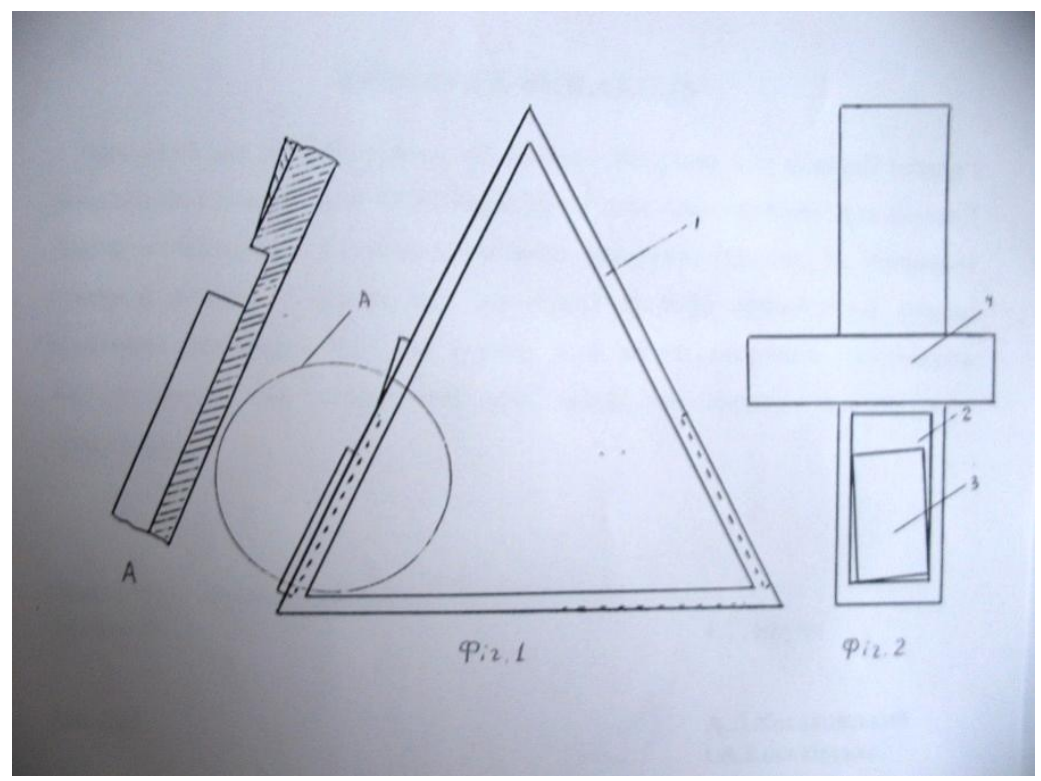

Fig. 3.2. Cartilage dissection device.

The device has the shape of a regular triangular prism (Fig. 1), on the outer surface of each of the faces (1) of which a rectangular groove (2) of various depths (0.5, 0.8 and 1 $\mathrm{mm})$ is made.

The device is used as follows.

The removed cartilage (3) is placed in the groove (2) in such a way that the lower edge of the cartilage is pressed against the lower wall of the groove, and the inner surface touches the bottom of the groove. The area of the cartilage is smaller than the area of the groove, therefore, to fix the cartilage, it is placed so that the lateral edges touch the side walls of the groove. This is achieved by rotating the cartilage in the groove.

To dissect the cartilage, a knife (3) made in the form of a right-angled triangle is used, which is placed on the working edge of the prism, tightly pressing against it and moved towards the cartilage.
Since the device has the shape of a regular triangular prism, placing it with one side on a horizontal surface, the working surface will be located at an angle of 600 and this ensures reliable fixation of the cartilage to the bottom of the groove during its opening due to gravity.

The length of the knife blade, which significantly exceeds the height of the facet of the prism and its shape in the form of a rightangled triangle, makes it possible to fix in one plane and thereby obtain plates of equal thickness throughout, which is equal to the depth of the groove. The choice of this or that face of the prism, as a working one, depends on the thickness of the cartilage plate and the specified thickness of the plate that will be manufactured.

For clarity, we present the results of the manufacture of plates using various tools (table 3.1). The thickness and shape of the 
cartilage were selected similarly in both cases. The difference was that in the first case, a scalpel and a spatula (basic object) were used to make cartilage plates, and in the second, the proposed device was used.

\section{Table 3.1}

The results of the manufacture of cartilage plates using various instruments

\begin{tabular}{|c|c|c|c|}
\hline Indicators & $\begin{array}{c}\text { Prototype } \\
\text { (base } \\
\text { object) } \\
\mathrm{n}=80\end{array}$ & $\begin{array}{c}\text { The proposed } \\
\text { device } \\
\mathrm{n}=80\end{array}$ & $\mathrm{P}$ \\
\cline { 1 - 3 } Plate making time (sec.) & $35 \pm 1,232$ & $7 \pm 0,411$ & $<0,01$ \\
\hline Cartilage plate manufacturing quality: & & 10 & \\
\hline - flat plate surface & 10 & 10 & \\
\hline - uneven plate surface & 1 & - & \\
\hline - the thickness of the plate is the same throughout & 9 & & \\
\hline - the thickness of the plate is not the same throughout & 4 & 10 & \\
\hline - the plate breaks easily when bent & 6 & & \\
\hline - the plate retains elasticity when bending & & & \\
\hline
\end{tabular}

From table 3.1 it can be seen that the time for making a plate from cartilage, when using the proposed device, decreases 5 times. In addition, the plates are made of significantly higher quality, namely: with a flat surface, of the same thickness, while maintaining the elasticity of the cartilage when it is bent.
The resulting thin and equal in area plates (from the removed cartilage) were implanted in place of the removed quadrangular cartilage and removed bony structures of the nasal septum: a ploughshare, the lower part of the perpendicular plate of the ethmoid bone, the crest of the palatine process of the upper jaw (Fig. 3.3). 


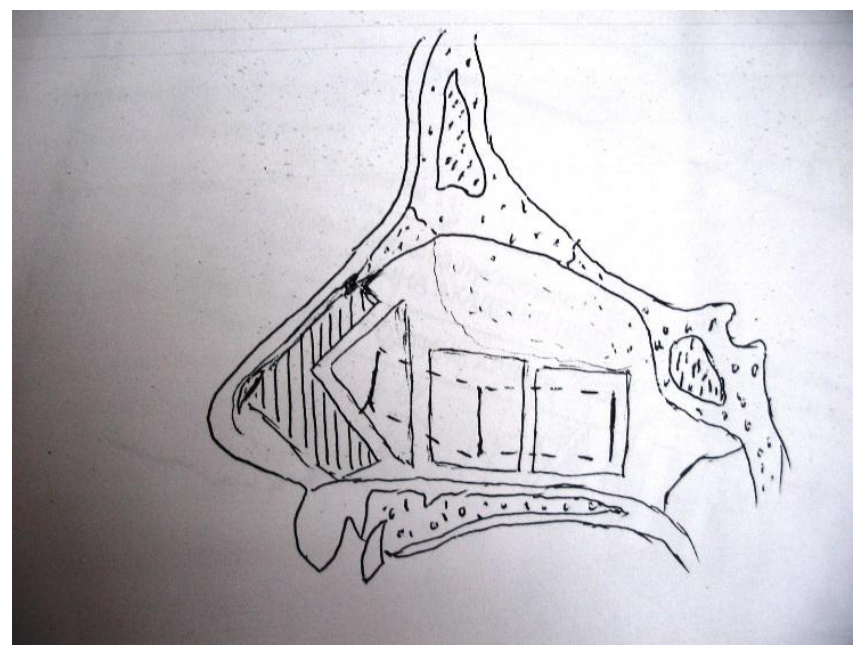

Fig. 3.3. Method of septoplasty with reimplantation of cartilage plates.

When the thickness of the quadrangular cartilage was insufficient to restore the elasticity of the nasal septum in the bony part, an oval-shaped plate was cut out on the lower leg in the posterior region of the cartilage and moved to the place of the removed ploughshare (Fig. 3.4).

Fig. 3.4. Method of septoplasty with cartilage displacement on the pedicle.

The reimplanted cartilage plates were fixed with U-shaped catgut or vicryl sutures in such a position to achieve contact with the bone. At the same time, the experimental studies of Melanin were taken into account, which showed that if a cartilage graft placed under the periosteum and affects the bones of the recipient, then it grows together with it, stimulates osteogenesis and is replaced by bone tissue. 
The curvature of the crest of the palatine process of the upper jaw was eliminated using

a drill (Fig. 3.5).

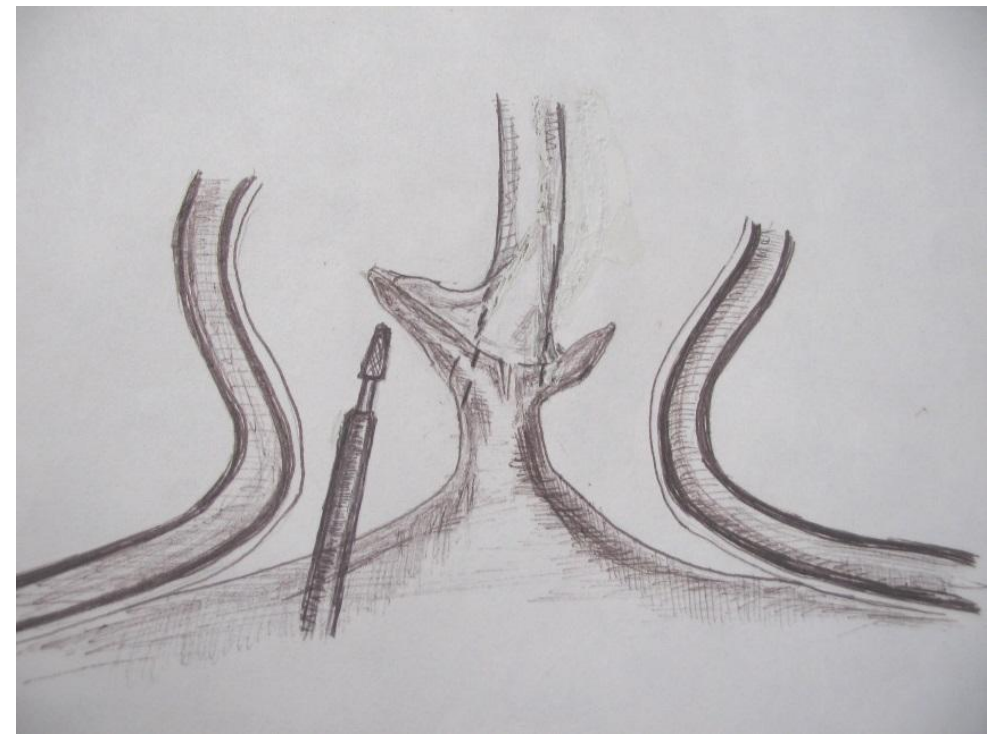

Fig. 3.5. The scheme for eliminating bone deformity in the anterior part of the nasal septum.

At the same time, special attention was paid not to damage the a.incisivae canal. In a number of cases, the connections between the quadrangular cartilage and the crest of the palatine process of the upper jaw were preserved, in others, before the elimination of the bone curvature, the quadrangular cartilage was mobilized along the lower edge.

In one case, the curvature of the nasal septum was due to an atypical position of the tooth. Here is a description of this case.

Child B., 8 years old, was admitted to the ENT department on 11/12/2017 with complaints of difficulty in nasal breathing, frequent bleeding from the left side of the nose for a month. The general condition was not disturbed. With anterior rhinoscopy, a curvature of the nasal septum on the left in the form of a thorn was diagnosed, which was localized in the anterior part of the nasal cavity at a distance of $1.5 \mathrm{~cm}$ from the entrance to the nose and $0.5 \mathrm{~cm}$ from the bottom of the nasal cavity and rested against the inferior turbinate. The mucous membrane of the thorn was thickened and had vascular injection.

During the operation, after detachment of the mucous membrane together with the perichondrium and periosteum from the lower sections of the nasal septum, it was revealed that the thorn is a tooth, the root of which is located in the bony ridge of the palatine process of the upper jaw under the quadrangular cartilage of the nasal septum. The distal end of the tooth was directed up and to the left towards the left inferior 
turbinate and was completely covered with mucous membrane.

The tooth was removed with the help of an elevator (Fig. 3.6). Since the mucous membrane that covers the tooth was thickened and vascular injection took place, as well as taking into account the history of nosebleeds, part of the mucous membrane was removed and sent for histological examination, and the edges of the mucous membrane were sutured with catgut.

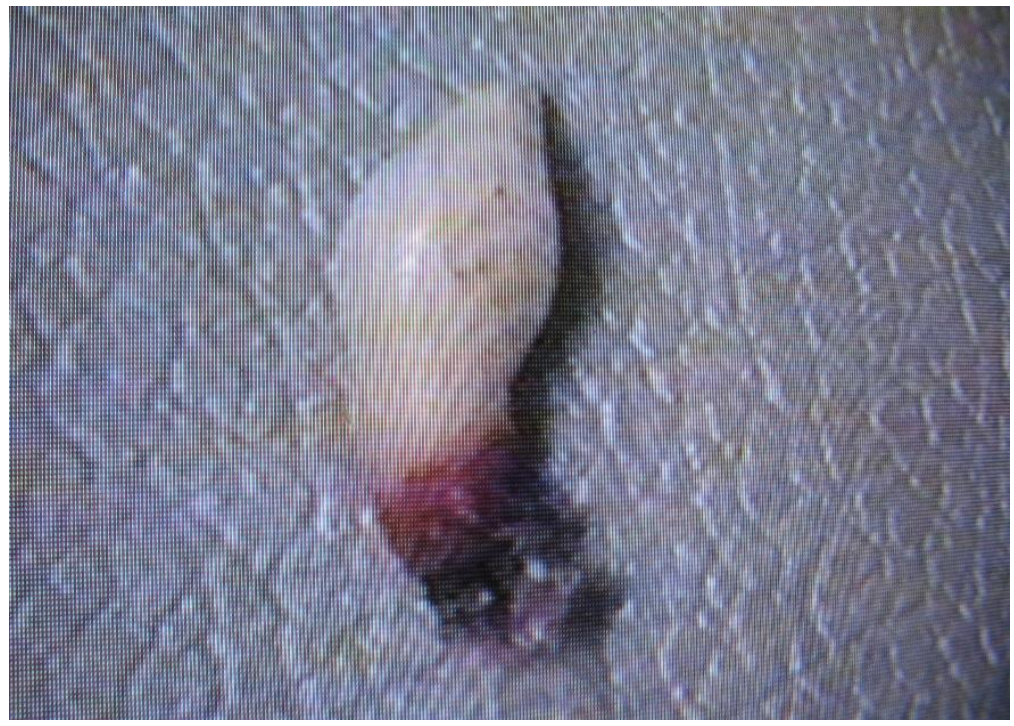

Fig. 3.6. An extracted tooth that is located in the thickness of the nasal septum.

According to the results of histological examination, the mucous membrane was a fibrous tissue with a large number of vessels and lymphoid infiltration, covered with stratified squamous epithelium.

As a result of the operation, the curvature of the nasal septum was eliminated, the patency of the nasal cavity was restored, and the nosebleeds stopped.

The peculiarity of this case is that the curvature of the nasal septum was due to the atypical location of the tooth and required a different approach to treatment.

Correction of curvature of the cartilaginous part of the nasal septum.
In the presence of curvature of the nasal septum in the cartilaginous part, the quadrangular cartilage during the operation was maximally preserved, removing only curved areas.

So, with a lumpy deformity, the cartilage was thinned, removing excess tissue while preserving the cartilage, which is located in the sagittal plane, drawn along the midline.

When the cartilage deformity was significant in a limited area in the form of a thorn or a small ridge, a part of the cartilage located outside the midline was removed. At the same time, sometimes in this area, after removing the cartilage, a small hole was formed, which did not affect the results of treatment, since the integrity of the mucous membrane was 
not excited during the operation, and the cartilage was almost completely preserved along the midline and provided elasticity of the nasal septum.

In most cases, cartilage curvature was localized in the lower part of the septum. In this case, only a part of the cartilage was removed, which deviated significantly from the midline. Since this deformity, as a rule, was combined with a significant curvature of the crest of the palatine process of the upper jaw, it was simultaneously thinned with preservation of the bone along the midline.

In some cases, it was possible to maintain the connection between the bone and cartilage,
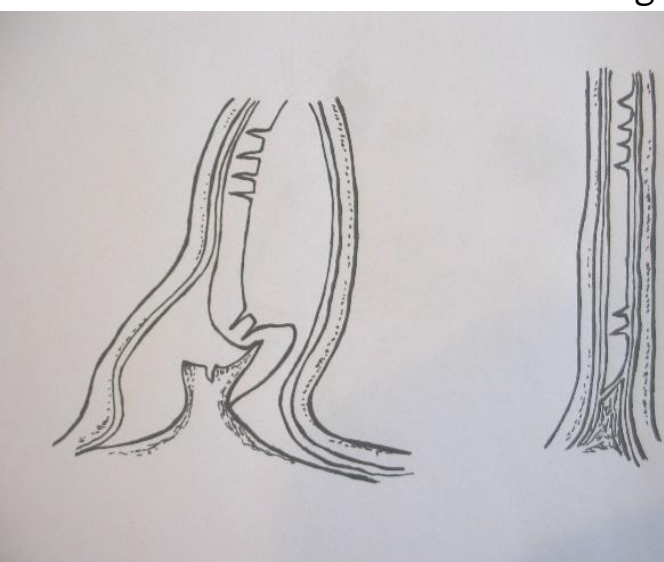

but in most cases it was necessary to mobilize the cartilage along the lower edge.

To prevent possible deformation of the nasal septum in the long-term postoperative period associated with displacement of the lower portion of the quadrangular cartilage, after eliminating the deformation of the quadrangular cartilage, we fixed its lower portion to the ridge of the palatine process of the upper jaw using sutures. In this case, on the concave side of the cartilage, parallel sections were applied to 2/3 of its thickness, and on the convex side, mucoperichondria peeling was avoided if possible (Fig. 3.7).

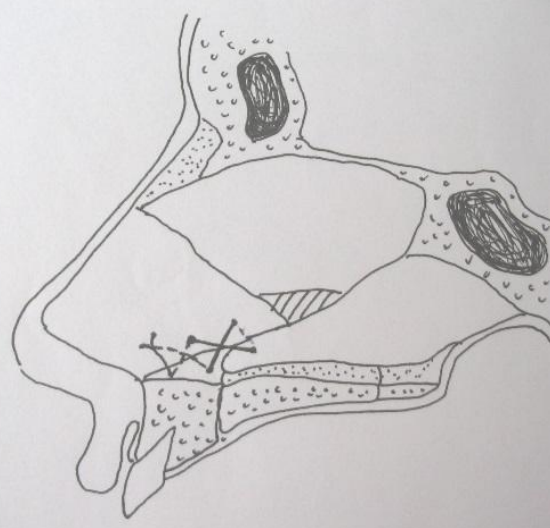

Fig. 3.7. Method of septoplasty for arcuate deformation of the cartilage of the nasal septum and in the form of a ridge in its lower part.

It is the preservation of the perichondrium on the convex side that facilitates the alignment of the cartilage $(71,192)$. When the mucoperichondria is detached from both sides, the straightening of the cartilage is not observed, and when mucoperichondria is detached, only on the convex side is an even greater deformation of the cartilage. The author argues that it is the perichondrium that plays the main role in the biomechanics of the cartilage and, during tension, contributes to its alignment.

In some cases, when the quadrangular cartilage was so deformed that it was not possible to preserve it, after its removal, except for strips $1 \mathrm{~cm}$ wide in the caudal region and $0.5 \mathrm{~cm}$ at the nasal dorsum, a plate was formed from the removed parts of the 
cartilage using interrupted sutures, which was reimplanted in place of the removed cartilage (mass $3.8 \mathrm{a}$ ), and in the case of its removal in one block, the curvature of the cartilage was

a

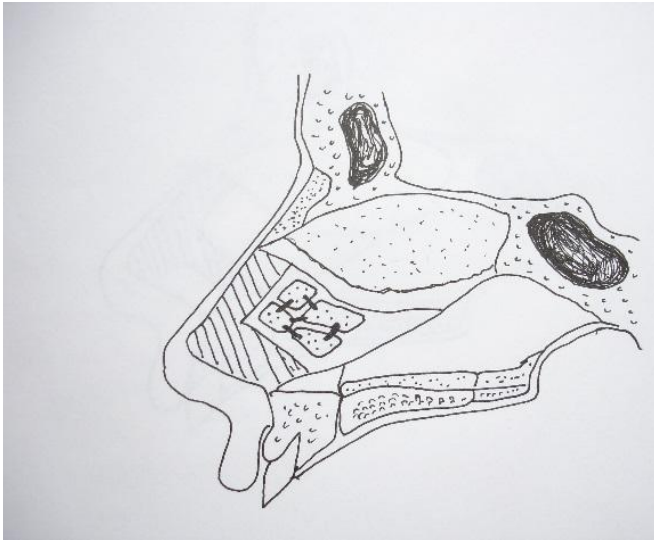

eliminated by flattening it, after which it was reimplanted (Fig. 3.8 b).

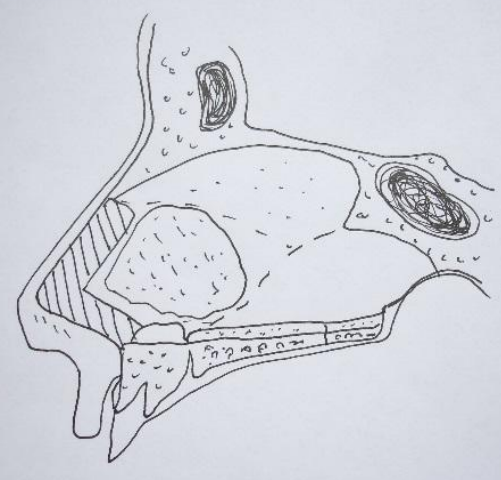

b

Fig. 3.8. Scheme of cartilage reimplantation after suturing individual fragments (a) and flattening it by flattening (b).

In the presence of subluxation of the quadrangular cartilage, the tactics of surgical treatment depended on the degree and form of its deformity. In patients with a slight deviation of the cartilage in the anterior part, its resection was performed in an area of several millimeters. In doing so, we took into account the recommendations of G.I. Piskunov and S.I. Piskunov and kept the resistance of the columella. In case of curvature of the quadrangular cartilage in only one plane (deviation to the right or left), the operation was performed according to the authors' technique (vertical section along the curvature line, removal of a narrow strip, transfer of the cartilage to the sagittal plane and fixation with sutures). With more pronounced deformity, the cartilage was removed in the anterior section with a width of 5 to $10 \mathrm{~mm}$ (in older children), and a straight cartilaginous plate taken from the posterior sections of the quadrangular cartilage was reimplanted in the place of the removed section (Fig. 3.9 a). In some cases, when there was a deformation of the twisted portion of the cartilage only in the middle and lower parts of it, resection of the cartilage was performed while maintaining the strip in the upper part of at least $5 \mathrm{~mm}$ wide, and to maintain the support of the columella, a cartilaginous plate taken from the parts of the quadrangular cartilage (Fig. 3.9 b). 


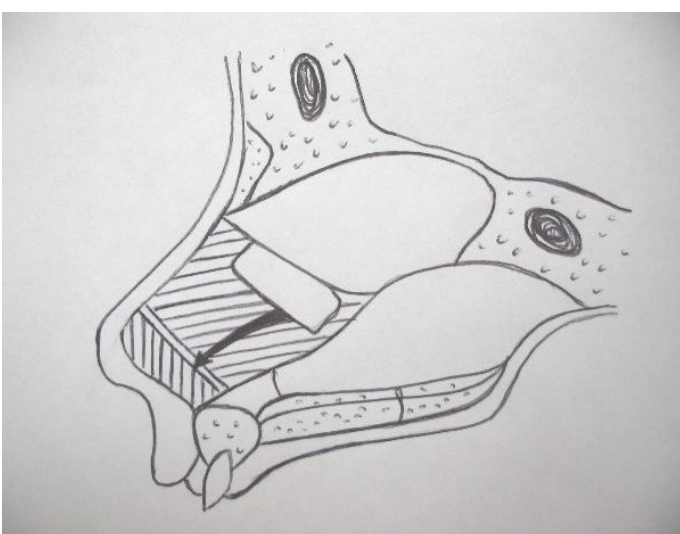

a

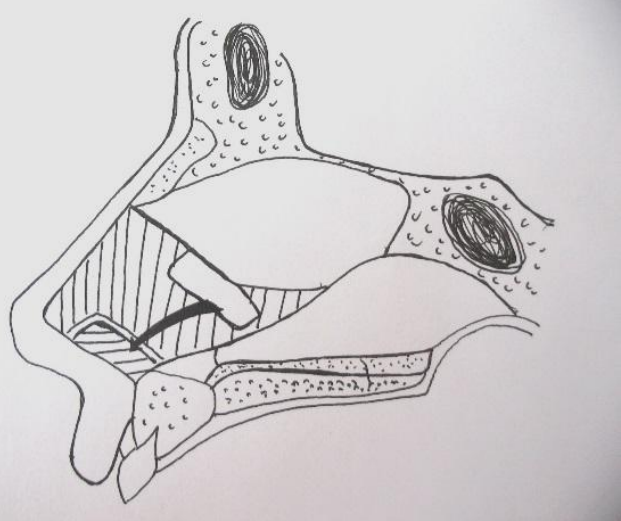

$b$

Fig. 3.9. Septoplasty modifications in case of "subluxation" of the quadrangular cartilage.

In one case, when the anterior part of the quadrangular cartilage was curved at an angle of $180^{\circ}$ and the cartilage itself was excessively elastic, the cartilage was dissected in the sagittal plane along the midline to create adequate support for the tip of the nose and maintain support for the columella, and then an additional plate was formed. cartilage up to $5 \mathrm{~mm}$ in width, which was hemmed with $\mathrm{U}$ shaped catgut sutures to the anterior section of the quadrangular cartilage (Fig. 3.10). As a result, the thickness of the anterior part of the cartilage was doubled and its deformities were eliminated. During the operation, the size and shape of the nasal valve was taken into account.

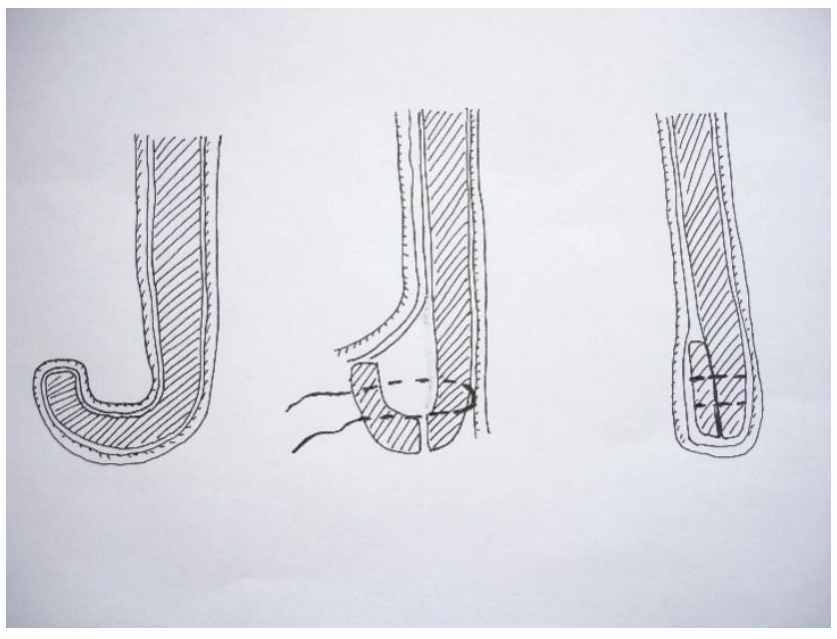

Fig. 3.10. Method of septoplasty for arcuate deformation of the anterior quadrangular cartilage (horizontal section). 
It should be noted that when isolating the quadrangular cartilage, its biomechanics were taken into account and the mucoperichondria were always exfoliated from the concave side and tried to preserve it on the convex side of the cartilage.

In order to prevent the formation of hematoma of the nasal septum in the postoperative period, careful hemostasis was performed, if necessary, using a diathermocoagulator. After cleaning the postoperative cavity on the mucous membrane of the nasal septum (from the side of the perichondrium and periosteum), step by step, starting from the posterior areas, Katsil glue was applied in the form of small drops at a distance of 7-10 mm from each other, followed by sequential comparison of the areas of the mucous membrane with each other or to the bone base and quadrangular cartilage and fixing them in a given position for 20-30 seconds (Fig. 3.11).

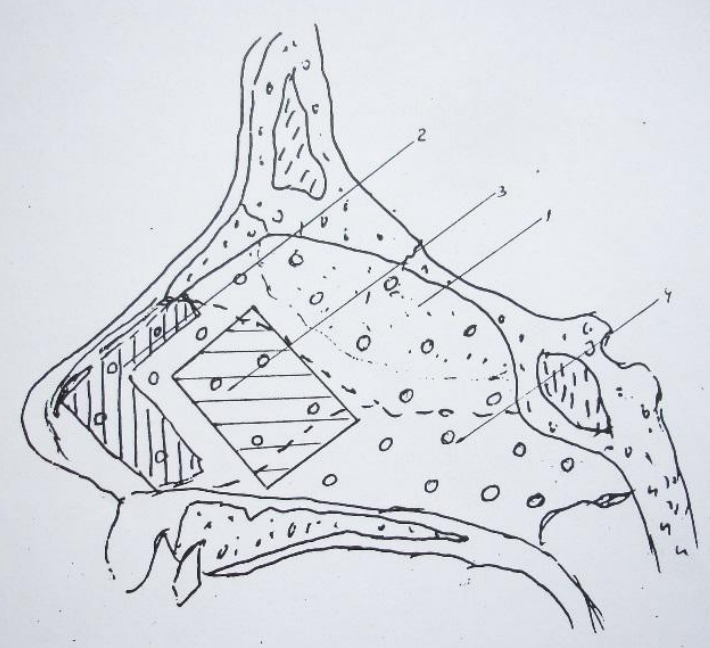

Fig. 3.11. Septoplasty method using "Katsil" glue.

In fig. 3.11 shows the scheme of applying Katsil glue. Drops of glue are applied to the bony part of the nasal septum (1), the preserved part of the quadrangular cartilage (2), the reimplanted cartilaginous plate (3) and the perichondrium and periosteum (4).
In some cases, when there was increased bleeding in the postoperative cavity and it was not possible to fix the mucous membrane sheets with glue, a round hole (2) with a diameter of 3-5 mm was made in one of the mucous membrane sheets (1), after which the mucous membranes the shells were fixed to each other with U-shaped seams (3) (Fig. 3.12). 


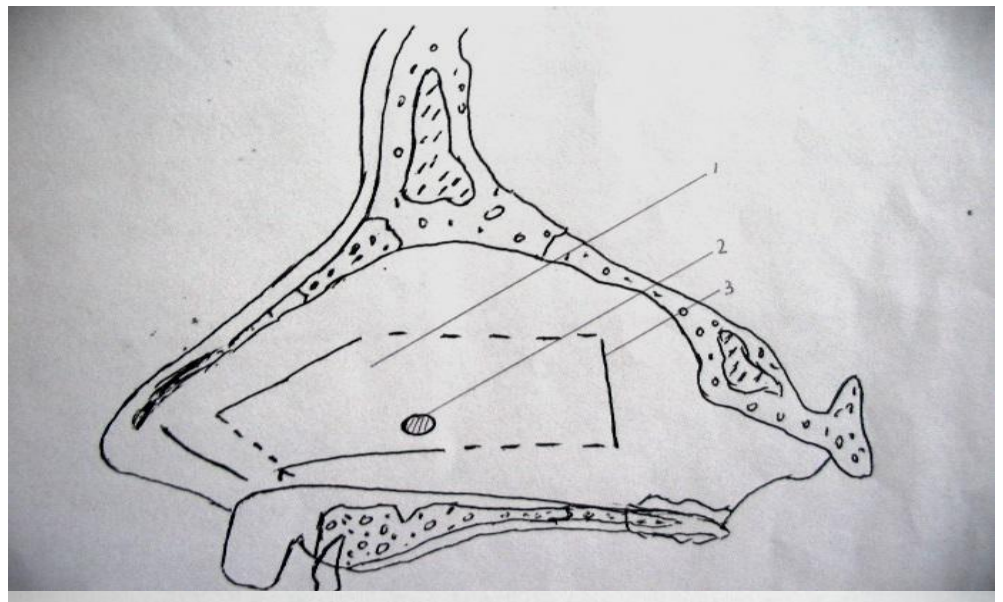

Fig. 3.12. Method for fixing mucous membrane sheets during septoplasty with increased bleeding.

A device by A.L. Kosakovsky or a straight needle with an arcuate curved proximal end. The latter has advantages, since, unlike needles with an arcuate bend, including at the distal end, the channel during the passage of the needle has not arcuate, but a rectilinear shape, which is especially important for fixing the cartilage in a given position. Unlike straight needles, the needle of this design allows you to make sutures not only in the front, but also in the back region of the nasal septum.

In 32 patients aged 12-47 years at the beginning of the study, due to severe deformity of the nasal septum, we had to perform a partial submucosal resection of the nasal septum.

At the end of the operation, anterior tamponade of the nasal cavity was performed. In this case, we used both traditional gauze 
swabs with $1 \%$ synthomycin liniment and swabs with hydroxylated polyvinyl acetate sponge ("Merocel” and "Entocel”).

In $16(14.6 \%)$ patients, the curvature of the nasal septum was combined with deformity of the external nose. In 14 cases, after the completion of septoplasty, rhinoplasty was performed using the closed technique.

In saddle deformity, the nasal dorsum was accessed through the anterior nasal septum. Using scissors, a tunnel was made under the skin, into which a plate (in most cases threelayered) made of quadrangular cartilage was subsequently placed. In one case, a cartilage implant was made from protocrill to correct the saddle deformity of the external nose. After the anterior section, the correct shape of the external nose was modeled and an external fixation bandage was applied.

The nasal tampons and outer dressing were removed at 5-6 days. During this time, patients were prescribed antibiotics.

In patients with scoliosis, deformity was eliminated after septoplasty and anterior nasal tamponade by mobilizing the bones of the external nose using the osteotomy method.

If it was necessary to use acute osteotomes, especially in case of tuberous deformity, access to the site of bone mobilization was performed through the lower Cottle tunnel or under the upper lip. The nasal dorsum was usually accessed transseptally in the anterior nasal septum.

\section{CONCLUSION}

Thus, when performing septoplasty in patients, it is advisable to use a tissuepreserving method of operations with preserving the skeleton of the nasal septum. After the elimination of the deformity of the nasal septum, when there was a displacement of the quadrangular cartilage, and its transfer to the median position, it is advisable to fix the cartilage to the bony part of the nasal septum along its lower edge, which contributes to its stabilization in the sagittal plane and allows to prevent the deviation of the cartilage from the median position to the postoperative one. period. The manufacture of cartilaginous plates to replace defects in the skeleton of the nasal septum is advisable to perform with the proposed device, which significantly reduces the time and improves their quality.

\section{REFERENCES}

1. Botirov, A. J., et al. "Clinical and morphological results of xenografts to use in myringoplasty." The International Tinnitus Journal 24.1 (2020): 1-6.

2. Djuraev, Jamolbek Abdukaxorovich, et al. "Results of Allergological and Immunological Research in Patients with Polipoid Rhinosinusitis." Asian Journal of Immunology (2020): 34-40.

3. Djuraev, Jamolbek Abdukhahharovich, Ulugbek Saidakramovich Khasanov, and Ulugbek Nuridinovich Vokhidov. "The prevalence of chronic inflammatory diseases of the nose and paranasal sinuses in patients with myocarditis." European Science Review 5-6 (2018): 147-149.

4. Normurodov, Bakhtoyor K., et al. "Prevalence and structure of purulent inflammatory diseases of the maxillofacial area." Central Asian 
Journal of Medicine 2020.1 (2020): 116130.

5. Jumanov, Daulet Azadbek Ugli, et al. "International Journal of Biological and Pharmaceutical Sciences Archive." International Journal of Biological and Pharmaceutical Sciences Archive 1.1 (2021): 011-015.

6. Djuraev, J. A., et al. "Results of an immunogistochemical study in patients with polipoid rhinosinusitis." European Journal of Molecular \& Clinical Medicine 7.2 (2020): 2526-2541.

7. Khasanov, U. S., and J. A. Djuraev. "Morphological characteristics of chronic polypous rhinosinusitis." cutting edge-science (2020): 30.

8. Shaumarov, A. Z., et al. "Role of Hemostatic Agents in Simultaneous Surgical Interventions in the Nasal Cavity."

9. Kurbonov, Yokubjon Khamdamovich, Shukhrat Abdujalilovich Boymuradov, and Jamolbek Abdukakharovich Djuraev. "Purulent-Necrotic Diseases Of The Face: Aspects Of Diagnostics And Treatment." The American Journal of Medical Sciences and Pharmaceutical Research 3.01 (2021): 24-30.

10. Narmurotov, Bakhtiyar Karshievich, Shukhrat Abdujalilovich Boymuradov, and Jamolbek Abdukakhkhorovich Djuraev. "Comparative Characteristics Of Rheological Properties Of Blood In Combined Face Injuries Before And After Treatment." The American Journal of Medical Sciences and Pharmaceutical Research 3.01 (2021): 67-75.

11. VOHIDOV, Ulugbek Nuridinovich, et al. "Current issues of the treatment of chronic polypous rhinosinusitis." Journal of Biomedicine and Practice 2.5 (2020).

12. Kurbonov, Yokubjon Khamdamovich, Shukhrat Abdujalilovich Boymuradov, and Jamolbek Abdukakharovich Djuraev. "Overview Of Comprehensive Treatment of Acute PurulentInflammatory Diseases of The Face And Neck." The American Journal of Medical Sciences and Pharmaceutical Research 3.01 (2021): 15-23.

13. Nigmatov, Iftikhor Obidjonovich, et al. "Post-Traumatic Defects And Face Deformations: Features Of Diagnostics And Treatment." The American Journal of Medical Sciences and Pharmaceutical Research 3.01 (2021): 55-66.

14. Djuraev, J. A. "Improvement of comprehensive treatment vasomotor rhinitis."

15. Khasanov, U. S., U. N. Vokhidov, and J. A. Djuraev. "State of the nasal cavity in chronic inflammatory diseases of the nose and paranasal sinuses in patients with myocarditis." European science,(9 (41)).-2018 (2018).

16. Vohidov, U. N., and J. A. Djuraev. "ugli, Makhsitaliev, MI, \& Khamidjanov, s." (2020).

17. UN, Vokhidov, et al. "The local immunity in the tissues of various forms of nasal polyps." ALLERGY. Vol. 71. 111 RIVER ST, HOBOKEN 070305774, NJ USA: WILEY-BLACKWELL, 2016.

18. Vohidov, U. N. "Djuraev JA ugli, Makhsitaliev, MI, \& Khamidjanov, s. O.(2020). Current issues of the treatment of chronic polypous 
The American Journal of Medical Sciences and Pharmaceutical Research

(ISSN - 2689-1026)

Published: February 20, 2021 | Pages: 13-30

IMPACT FACTOR

2021: 5.64

Doi: https://doi.org/10.37547/TAJMSPR/Volume03Issue02-03

OCLC - 1121105510

rhinosinusitis." Journal of Biomedicine and Practice 2.5.

19. Makhsitaliev, Mukhammadbobur, et al. "The Functional State of The Mucous Membrane of The Nasal Cavity And Paranasal Sinuses After Radical And Minimally Invasive Surgical Interventions." The American Journal of Medical Sciences and Pharmaceutical Research 3.01 (2021): 31-40. 